\title{
Advanced Practice Nurse Practitioner
}

National Cancer Institute

\section{Source}

National Cancer Institute. Advanced Practice Nurse Practitioner. NCI Thesaurus. Code C71285.

A registered nurse with advanced education, knowledge, skills, and scope of practice. 